\title{
The Poisson Kernel for Hardy Algebras
}

\author{
Paul S. Muhly* \\ Department of Mathematics \\ University of Iowa \\ Iowa City, IA 52242 \\ e-mail: muhly@math.uiowa.edu \\ Baruch Solel ${ }^{\dagger}$ \\ Department of Mathematics \\ Technion \\ 32000 Haifa, Israel \\ e-mail: mabaruch@techunix.technion.ac.il
}

October 26, 2018

\section{Introduction}

This note contributes to a circle of ideas that we have been developing recently in which we view certain abstract operator algebras, which we call Hardy algebras, and which are noncommutative generalizations of classical $H^{\infty}$, as spaces of functions defined on their spaces of representations [11, 12, 13, 14]. This perspective leads to a number of pleasant formulas that are very reminiscent of formulas from complex function theory on the unit disc. More important, however, they help to reveal structural properties of the algebras and they help to clarify the interplay among various constructs that are at work in their analysis. Even in the classical setting of complex functions of one variable, insight is sometimes gained by viewing classical $H^{\infty}$ as a space of functions on its space of representations, which are parameterized, essentially, by all the completely non-unitary contractions. Another source of motivation is the work of Popescu, Davidson and Pitts, and others who have done extensive work on free semigoup algebras 1 Indeed, many of the results that we prove here have been been anticipated in this work. What is novel about our approach, however,

\footnotetext{
* Supported in part by grants from the National Science Foundation and from the U.S.Israel Binational Science Foundation.

${ }^{\dagger}$ Supported in part by the U.S.-Israel Binational Science Foundation and by the B. and G. Greenberg Research Fund (Ottawa).

${ }^{1}$ For a nice survey of the basics of free semigroup algebras, we recommend Ken Davidson's article [5].
} 
is the systematic use of "duality of correspondences" to put into evidence the effectiveness of viewing elements of our Hardy algebras as functions on operator discs. When this is done, proofs in the free semigroup picture often become simpler, shorter and more perspicuous. And they extend to a wide variety of additional situations in the literature that are of interest.

In the next section, we introduce the basic players in our theory: a $W^{*}$ algebra $M$, a $W^{*}$-correspondence $E$ over $M$ and the Hardy algebra they generate: $H^{\infty}(E)$. We then describe how a normal representation $\sigma: M \rightarrow B\left(H_{\sigma}\right)$ gives rise to a "dual" correspondence, denoted $E^{\sigma}$ and we describe how elements of $H^{\infty}(E)$ may be realized as functions defined on the unit ball of the space of adjoints of $E^{\sigma}, \mathbb{D}\left(\left(E^{\sigma}\right)^{*}\right)$. In Section 3, we define a generalization of the Poisson kernel, which "reproduces" the values on $\mathbb{D}\left(\left(E^{\sigma}\right)^{*}\right)$ of the "functions" coming from $H^{\infty}(E)$. When $M=E=\mathbb{C}$ and $\sigma$ is the one dimensional representation of $M$, then $H^{\infty}(E)$ is classical $H^{\infty}$ realized as analytic Toeplitz operators, and our Poisson kernel is easily seen to be the classical Poison kernel formulated in terms of operators on Hilbert space. Our representation theorems, Theorems 12 and 14 are easily seen to be natural generalizations of the Poisson integral formuala. They also are easily seen to be generalizations of formulas that Popescu developed in [15] and elsewhere, and they are closely related to formulas that Arveson developed in [2]. In the fourth section, we relate our Poisson kernel to the idea of a characteristic operator function and show how the Poisson kernel identifies the "model space" for the canonical model that can be attached to a point in the disc $\mathbb{D}\left(\left(E^{\sigma}\right)^{*}\right)$ - a structure we developed in [12. We were inspired here by [17] and other results from literature. In the next section, Section 5, we consider a Poisson kernel on the unit ball of $E, \mathbb{D}(E)$. Owing to our duality theorem [11, Theorem 3.9], one can think of this ball as the place to evaluate elements in $H^{\infty}\left(E^{\sigma}\right)$, but in addition, it captures ideas about "(left) point evaluations" that appear in the systems theory literature, cf. 11. Finally, in Section 6 , we connect our Poission kernel to the idea of curvature and complement results that we proved in [9]. Again, our analysis extends parts of the theory of curvature for not-necessarily-commuting row contractions that was developed by Popescu in [16]. His work, in turn, was based on investigations by Arveson [2]

in which he introduced a notion of curvature to study properties of commuting row contractions.

\section{Preliminaries}

We recall a few key ideas from [11] and we refer to that paper for further discussion and references about the setup with which we will be working here. Throughout this note $M$ will be a fixed $W^{*}$-algebra. We also fix a $W^{*}$-correspondence $E$ over $M$. This means that $E$ is a self-dual Hilbert $C^{*}$-module over $M$ and that there is a normal homomorphism $\varphi$ from $M$ into the $W^{*}$-algebra of all continuous module maps on $E, \mathcal{L}(E)$, giving $E$ an action of $M$ that makes $E$ a bimodule over $M$. We shall form the (balanced) tensor powers of $E, E^{\otimes n}$, which are all $W^{*}$-correspondences over $M$, and we shall denote the left action 
of $M$ on $E^{\otimes n}$ by $\varphi_{n}$. It is defined by the formula

$$
\varphi_{n}(a)\left(\xi_{1} \otimes \xi_{2} \otimes \cdots \otimes \xi_{n}\right)=\left(\varphi(a) \xi_{1}\right) \otimes\left(\xi_{2} \otimes \cdots \otimes \xi_{n}\right) .
$$

We shall write $E^{\otimes 0}=M$, viewed as a bimodule over itself, so in particular, $\varphi_{0}(a) \xi=a \xi$. The direct sum $E^{\otimes 0} \oplus E^{\otimes 1} \oplus E^{\otimes 2} \oplus \cdots$ is a $W^{*}$-correspondence over $M$ in an obvious and natural way, which we shall denote by $\mathcal{F}(E)$ and call the Fock space over $E$. The left action of $M$ on $\mathcal{F}(E)$ is the sum of the $\varphi_{n}$ and will be denoted $\varphi_{\infty}$. Thus, for $a \in M$,

$$
\varphi_{\infty}(a)=\operatorname{diag}\left(\varphi_{0}(a), \varphi_{1}(a), \varphi_{2}(a), \ldots\right),
$$

when we view operators as matrices on $\mathcal{F}(E)$ as we shall. An element $\xi \in E$ defines a creation operator $T_{\xi}$ on $\mathcal{F}(E)$ via the formula $T_{\xi} \eta=\xi \otimes \eta$. This operator is bounded, with adjoint given by the formula $T_{\xi}^{*}(\zeta \otimes \eta)=\varphi_{\infty}(\langle\xi, \zeta\rangle) \eta$. Matricially, $T_{\xi}$ has a form of an operator-valued weighted shift:

$$
T_{\xi}=\left[\begin{array}{ccccc}
0 & & & & \\
T_{\xi}^{(1)} & 0 & & 0 & \\
0 & T_{\xi}^{(2)} & 0 & & \\
& 0 & T_{\xi}^{(3)} & \ddots & \\
0 & & \ddots & \ddots & \ddots
\end{array}\right]
$$

where $T_{\xi}^{(n)}$ maps $E^{\otimes(n-1)}$ into $E^{\otimes n}$ by tensoring with $\xi$. The ultraweakly closed subalgebra of $\mathcal{L}(\mathcal{F}(E))$ generated by the $T_{\xi}, \xi \in E$, and the $\varphi_{\infty}(a), a \in M$, is called the Hardy algebra of $E$ and is denoted $H^{\infty}(E)$. Numerous examples of Hardy algebras may be found in the literature that we cite, and elsewhere, so we won't go into detail here. However, we do want to point out that when $M=E=\mathbb{C}$, the complex numbers, then $H^{\infty}(E)$ is the classical Hardy space of bounded analytic functions on the open unit disc, $H^{\infty}$, realized as the algebra of all (bounded) analytic Toeplitz operators on the space $\ell^{2}\left(\mathbb{Z}_{+}\right)$. Hence the terminology.

A fundamental feature of our theory is that the ultraweakly continuous completely contractive representations of $H^{\infty}(E)$ can be parametrized by the normal representations of $M$ and certain contraction operators in a fashion that we want to describe in some detail. Let $\sigma: M \rightarrow B(H)$ be a normal representation of $M$ on a Hilbert space $H$. Then $\sigma$ induces a normal representation $\sigma^{E}$ of $\mathcal{L}(E)$ on $E \otimes_{\sigma} H$, defined via the formula $\sigma^{E}(X)=X \otimes I_{H}$. In fact, $\sigma^{E}$ is called the induced representation of $\mathcal{L}(E)$ determined by $\sigma$, and we refer to [18] for a discussion of the general theory. If we form $\sigma^{E} \circ \varphi$ we obtain a new representation of $M$ that we denote simply by $\varphi \otimes I$ and refer to as the induced representation of $M$ determined by $\sigma$ (and $E$ ). Suppose that $T$ is an operator from $E \otimes_{\sigma} H$ to $H$ of norm at most one that intertwines the induced representation of $M$ and $\sigma$, i.e., suppose

$$
T\left(\varphi(a) \otimes I_{H}\right)=\sigma(a) T
$$


for all $a \in M$, then $T$ determines an ultraweakly continuous, completely contractive bimodule map $\hat{T}$ from $E$ to $B(H)$ via the formula

$$
\hat{T}(\xi) h=T(\xi \otimes h),
$$

$\xi \in E$ and $h \in H$. That is, $\hat{T}: E \rightarrow B(H)$ is completely contractive, where $E$ is regarded as an operator space in the operator space structure it inherits as a subspace of its linking algebra [6, Page 398], and is continuous with respect to the natural so-called $\sigma$-topology of [4] and the ultraweak topology on $B(H)$. The bimodule property refers to the equation $\hat{T}(\varphi(a) \xi b)=\sigma(a) \hat{T}(\xi) \sigma(b)$, which is satisfied for all $a, b \in M$ and $\xi \in E$. We call the pair $(\hat{T}, \sigma)$ an (ultraweakly continuous completely contractive) covariant representation of $E$ (and $M$ ) on $H$. Conversely, given such a representation of $E$ and $M$ on a Hilbert space $H$, $(S, \sigma)$, the formula

$$
\tilde{S}(\xi \otimes h):=S(\xi) h
$$

$\xi \otimes h \in E \otimes_{\sigma} H$ defines an operator of norm at most 1 from $E \otimes_{\sigma} H$ to $H$ that satisfies equation (1). We denote this operator by $\tilde{S}$, i.e., $\tilde{S}\left(\varphi(a) \otimes I_{H}\right)=\sigma(a) \tilde{S}$ for all $a \in M$. Clearly, we have $\tilde{\hat{T}}=T$ and $\hat{\tilde{T}}=T$.

The key point is that each ultraweakly continuous, completely contractive representation $\rho$, say, of $H^{\infty}(E)$ on a Hilbert space $H$ determines a completely contractive covariant representation of $E$ and $M$ on $H$ through the formulas

$$
\sigma(a)=\rho\left(\varphi_{\infty}(a)\right)
$$

and

$$
T(\xi)=\rho\left(T_{\xi}\right),
$$

and conversely, (almost) every completely contractive covariant representation $(T, \sigma)$ "integrates" to an ultraweakly continuous, completely contractive representation $\rho$ through these formulas. We say "almost" because while every $(T, \sigma)$ "integrates" to a norm-continuous, completely contractive representation $\rho$ of the norm-closed algebra generated by $\left\{T_{\xi}\right\}_{\xi \in E}$ and $\varphi_{\infty}(M)$, which we denote by $\mathcal{T}_{+}(E)$ and call the tensor algebra of the correspondence, the representation $\rho$ need not extend all the way to $H^{\infty}(E)$. (We will say more about this in a moment.) We write $\sigma \times T$ for the representation determined by $(T, \sigma)$ on the norm-closed algebra whether or not it extends to $H^{\infty}(E)$ 2. If $\|\tilde{T}\|<1$, then $\sigma \times T$ does extend to an ultraweakly continuous, completely contractive representation of $H^{\infty}$ [11, Corollary 2.14]. Thus we can say that once a normal representation $\sigma$ of $M$ on $H$ is given, then there is a bijective correspondence between the strictly contractive intertwiners of $\sigma$ and $\varphi \otimes I$ and the ultraweakly continuous, completely contractive representations $\rho$ of $H^{\infty}(E)$ on $H$ such that $\rho \circ\left(\varphi_{\infty} \otimes I_{H}\right)=\sigma$ and such that $\left\|\rho\left(T_{\xi}\right)\right\|<c\|\xi\|$ for all $\xi \in E$, where $c$ is a prescribed constant less than 1 . This observation suggests that we may adopt the perspective of viewing elements of $H^{\infty}(E)$ as functions on the space of (ultraweakly continuous, completely contractive) representations of $H^{\infty}(E)$ in a

\footnotetext{
${ }^{2}$ In some of our papers, we have written " $T \times \sigma^{\prime \prime}$ instead of " $\sigma \times T$ ". We apologize for any confusion this may create.
} 
concrete and transparent fashion. This suggestion was the principal point of [11 and has been the focus of much of our subsequent work. To help explain further the functional perspective initiated in [11], we require the following definition.

Definition 1 If $\sigma: M \rightarrow B(H)$ is a normal representation of $M$ on the Hilbert space $H$, then we define $E^{\sigma}$ to be the space of bounded operators $\eta: H \rightarrow E \otimes_{\sigma} H$ with the property that $\eta \sigma(a)=\left(\varphi(a) \otimes I_{H}\right) \eta$ for all $a \in M$. We call $E^{\sigma}$ the $\sigma$ dual of $E$. We write $\mathbb{D}\left(E^{\sigma}\right)$ for the open unit ball in $E^{\sigma}$.

Evidently, the elements of $E^{\sigma}$ are precisely the adjoints of the space of operators that satisfy equation (11). Suppose $\eta \in \mathbb{D}\left(E^{\sigma}\right)$ is given. Then $\eta^{*}$ satisfies equation (11) and determines an ultraweakly, completely contractive covariant representation $\left(\widehat{\eta^{*}}, \sigma\right)$ of $E$ on $H$. Further, with the aid of [11, Corollary 2.14], the formulas

$$
\varphi_{\infty}(a) \rightarrow \sigma(a)
$$

and

$$
T_{\xi} \rightarrow \widehat{\eta^{*}}(\xi)
$$

extend to give an ultraweakly continuous, completely contractive representation $\sigma \times \widehat{\eta^{*}}$ of $H^{\infty}(E)$ on $H$. On elements of the form $\left(T_{\xi_{1}} \otimes I_{H}\right)\left(T_{\xi_{2}} \otimes I_{H}\right) \cdots\left(T_{\xi_{n}} \otimes\right.$ $\left.I_{H}\right)=\left(T_{\xi_{1} \otimes \xi_{2} \otimes \cdots \xi_{n}} \otimes I_{H}\right)$, for example, $\sigma \times \widehat{\eta^{*}}$ is given by the formula

$$
\sigma \times \widehat{\eta^{*}}\left(T_{\xi_{1} \otimes \xi_{2} \otimes \cdots \xi_{n}} \otimes I_{H}\right)=\widehat{\eta^{*}}\left(\xi_{1}\right) \widehat{\eta^{*}}\left(\xi_{2}\right) \cdots \widehat{\eta^{*}}\left(\xi_{n}\right) .
$$

Following [12], we introduce the following terminology.

Definition 2 For $\eta \in \mathbb{D}\left(E^{\sigma}\right)$ and for $X \in H^{\infty}(E)$, we define

$$
\widehat{X}\left(\eta^{*}\right):=\sigma \times \widehat{\eta^{*}}(X) .
$$

The resulting function $\widehat{X}: \mathbb{D}\left(E^{\sigma}\right)^{*} \rightarrow B(H)$ is called the Fourier transform of $X$.

Perhaps the term " $Z$-transform" is preferable to "Fourier transform", but both conjure up formulas such as $\widehat{X Y}\left(\eta^{*}\right)=\widehat{X}\left(\eta^{*}\right) \widehat{Y}\left(\eta^{*}\right)$ that are clearly evident from (4).

Remark 3 Suppose $M=E=\mathbb{C}$ and that $H$ also is $\mathbb{C}$. Then of course $\sigma$ can only be the identity representation of $M=\mathbb{C}$ on $H, E^{\sigma}$ also may be identified with $\mathbb{C}$. In this situation, then, $\mathbb{D}\left(E^{\sigma}\right)$ is just the open unit disc $\mathbb{D}$ in the complex plane. The Fourier transform takes an $X$ in $H^{\infty}(E)$, which by our definition is an infinite, lower-triangular, Toeplitz matrix on $\ell^{2}\left(\mathbb{Z}_{+}\right)$

$$
X=\left(\begin{array}{ccccc}
a_{0} & 0 & 0 & \ldots & \ldots \\
a_{1} & a_{0} & 0 & \ddots & \ddots \\
a_{2} & a_{1} & a_{0} & 0 & \ddots \\
a_{3} & a_{2} & a_{1} & \ddots & \ddots \\
\vdots & \ddots & \ddots & \ddots & \ddots
\end{array}\right)
$$


that represents a bounded operator, to a function from $\mathbb{D}$ to operators on $H=\mathbb{C}$, i.e., to numbers. To compute them, simply note that for $\eta \in \mathbb{D}, \eta^{*}$ is just the complex conjugate of $\eta, \bar{\eta}$, and equation (5) implies that $\widehat{X}\left(\eta^{*}\right)$ is nothing but multiplication by the complex number $\sum_{k=0}^{\infty} a_{k} \bar{\eta}^{k}$ on $\mathbb{C}$, i.e., for $c \in \mathbb{C}, \widehat{X}\left(\eta^{*}\right) c=$ $\left(\sum_{k=0}^{\infty} a_{k} \bar{\eta}^{k}\right)$ c. It is clear in this example, that for no $\eta$ on the boundary of $\mathbb{D}$ does $\sigma \times \widehat{\eta}^{*}$ extend to an ultraweakly continuous representation of $H^{\infty}(E)$. If, next, $H=\mathbb{C}^{n}$, and again if $\sigma(a) \xi=a \xi$, for $a \in M=\mathbb{C}$, then $E^{\sigma}$ may be viewed as the $n \times n$ matrices over $\mathbb{C}$, and $\mathbb{D}\left(E^{\sigma}\right)$ consists of all those $n \times n$ matrices of norm less than 1 . If $T$ is such a matrix, then $\widehat{X}\left(T^{*}\right)$ is the operator on $H=\mathbb{C}^{n}$ given by a similar formula:

$$
\widehat{X}\left(T^{*}\right) \xi=\left(\sum_{k=0}^{\infty} a_{k} T^{*^{k}}\right) \xi
$$

It is clear in this case, that for $\|T\|=1, \sigma \times \widehat{T^{*}}$ extends to an ultraweakly continuous representation of $H^{\infty}(E)$ on $H$ if and only if the spectral radius of $T$ is less than one. Finally, if $H$ is an infinite dimensional Hilbert space, so that $\sigma(a) \xi=a \xi$, as before, then $E^{\sigma}$ may be identified with $B(H)$ and $\mathbb{D}\left(E^{\sigma}\right)$ may be viewed as the collection of all operators on $H$ of norm less than one. In this case, $\widehat{X}\left(T^{*}\right)$ again is given by the formula (6). Now, however, the $T$ 's of norm one for which $\sigma \times \widehat{T^{*}}$ extends to an ultraweakly continuous representation of $H^{\infty}(E)$ are precisely those whose minimal unitary dilations are absolutely continuous with respect to Lebesgue measure on the circle. Such a contraction splits into a completely non-unitary contraction and an absolutely continuous unitary operator. The value of $\widehat{X}\left(T^{*}\right)$ for such a $T$ is given by the Sz.-Nagy - Foias functional calculus. In [11, Section 7] we showed, in general, that if $\eta \in \mathbb{D}\left(E^{\sigma}\right)$ is such that $\eta^{*}$ is "completely noncoisometric", then $\sigma \times \widehat{\eta^{*}}$ extends to an ultraweakly continuous representation of $H^{\infty}(E)$. Beyond this, it is a mystery to us about how to identify points $\eta$ on the boundary of $\mathbb{D}\left(E^{\sigma}\right)$ in general such that $\sigma \times \widehat{\eta^{*}}$ extends to an ultraweakly continuous representation of $H^{\infty}(E)$.

The reason we focus on $E^{\sigma}$ rather than on the space of its adjoints, $\left(E^{\sigma}\right)^{*}$, at least for some purposes, is that $E^{\sigma}$ is a $W^{*}$-correspondence over the commutant of $\sigma(M), \sigma(M)^{\prime}$. The point to keep in mind is that the commutant of $\sigma^{E}(\mathcal{L}(E))$ is $I_{E} \otimes \sigma(M)^{\prime}$ [18, Theorem 6.23], and so $E^{\sigma}$ becomes a bimodule over $\sigma(M)^{\prime}$ according to the formula

$$
a \cdot \eta \cdot b:=\left(I_{E} \otimes a\right) \eta b,
$$

$a, b \in \sigma(M)^{\prime}, \xi \in E^{\sigma}$. The $\sigma(M)^{\prime}$-valued inner product on $E^{\sigma}$ is given simply by operator multiplication:

$$
\langle\eta, \zeta\rangle:=\eta^{*} \zeta
$$

$\eta, \zeta \in E^{\sigma}$. For more details about the structure of $E^{\sigma}$ and examples, see Sections 3 and 4 of [11.

One of the important points for us in this note is that for the representations $\rho$ of $H^{\infty}(E)$ that we defined in [7] and called induced representations, the 
commutant of $\rho\left(H^{\infty}(E)\right)$ can be expressed in terms of induced representations of $H^{\infty}\left(E^{\sigma}\right)$.

Definition 4 Let $\sigma: M \rightarrow B(H)$ be a normal representation of $M$ on a Hilbert space $H$ and form the Hilbert space $\mathcal{F}(E) \otimes_{\sigma} H$. The induced covariant representation of $E$ determined by $\sigma$ is the representation $\left(V, \varphi_{\infty} \otimes I_{H}\right)$ where $V: E \rightarrow B\left(\mathcal{F}(E) \otimes_{\sigma} H\right)$ is defined by the equation

$$
V(\xi)(\eta \otimes h):=(\xi \otimes \eta) \otimes h,
$$

$\xi \in E$, and $\eta \otimes h \in \mathcal{F}(E) \otimes_{\sigma} H$. The integrated form of $\left(V, \varphi_{\infty} \otimes I_{H}\right),\left(\varphi_{\infty} \otimes\right.$ $\left.I_{H}\right) \times V$, is called the representation of $H^{\infty}(E)$ induced by $\sigma$. We shall usually write $\sigma^{\mathcal{F}(E)}$ for $\left(\varphi_{\infty} \otimes I_{H}\right) \times V$, and most frequently, we will simply write $X \otimes I_{H}$ for $\sigma^{\mathcal{F}(E)}(X), X \in H^{\infty}(E)$.

The map $V$ is essentially the map defining the tensor powers of $E$ and the associated map $\tilde{V}: E \otimes(\mathcal{F}(E) \otimes H) \rightarrow \mathcal{F}(E) \otimes H$ appears to be just the identity map embedding $\sum_{k=1}^{\infty}\left(E^{\otimes k} \otimes_{\sigma} H\right)$ into $\mathcal{F}(E) \otimes_{\sigma} H$. However, it is a bit more complicated. There is a shift involved, as we shall see later in equation (24) and subsequent analysis.

Observe that if $\eta \in E^{\sigma}$, then for each $k \geq 0, I_{E \otimes k} \otimes \eta$ may be viewed as a map from $E^{\otimes k} \otimes_{\sigma} H$ to $E^{\otimes(k+1)} \otimes_{\sigma} H$. Further, due to the balanced nature of the tensor products,

$$
\left(I_{E^{\otimes k}} \otimes \eta\right)\left(\varphi_{k}(a) \otimes I_{H}\right)=\left(\varphi_{k+1}(a) \otimes I_{H}\right)\left(I_{E^{\otimes k}} \otimes \eta\right) .
$$

Consequently, we may define a map $U: \mathcal{F}\left(E^{\sigma}\right) \otimes_{\iota} H \rightarrow \mathcal{F}(E) \otimes_{\sigma} H$, where $\iota$ denotes the identity representation of $\sigma(M)^{\prime}$ in $B(H)$, so that on elements of the form $\eta_{1} \otimes \eta_{2} \otimes \cdots \eta_{n} \otimes h \in \mathcal{F}\left(E^{\sigma}\right) \otimes_{\iota} H, U$ is given by the formula

$$
U\left(\eta_{1} \otimes \eta_{2} \otimes \cdots \eta_{n} \otimes h\right)=\left(I_{E \otimes(n-1)} \otimes \eta_{1}\right)\left(I_{E \otimes(n-2)} \otimes \eta_{2}\right) \cdots\left(I_{E} \otimes \eta_{n-1}\right) \eta_{n} h .
$$

In [12], we called $U$ the (inverse) Fourier transform mapping $\mathcal{F}\left(E^{\sigma}\right) \otimes_{\iota} H$ to $\mathcal{F}(E) \otimes_{\sigma} H$ determined by $E$ and $\sigma$. It plays a fundamental role in our theory, as demonstrated by the following theorem, which is a restatement of parts of Lemma 3.8 and Theorem 3.9 of [11].

Theorem 5 Let $\sigma: M \rightarrow B(H)$ be a faithful normal representation of $M$ on the Hilbert space $H$. Then the inverse Fourier transform $U: \mathcal{F}\left(E^{\sigma}\right) \otimes_{\iota} H \rightarrow$ $\mathcal{F}(E) \otimes_{\sigma} H$ is a Hilbert space isomorphism such that the map

$$
X \rightarrow U \iota^{\mathcal{F}\left(E^{\sigma}\right)}(X) U^{*}
$$

from $H^{\infty}\left(E^{\sigma}\right)$ to $B\left(\mathcal{F}(E) \otimes_{\sigma} H\right)$ is an ultraweakly homeomorphic, completely isometric isomorphism from $H^{\infty}\left(E^{\sigma}\right)$ onto the commutant of $\sigma^{\mathcal{F}(E)}\left(H^{\infty}(E)\right)$. Likewise, the map

$$
X \rightarrow U^{*} \sigma^{\mathcal{F}(E)}(X) U
$$

is an ultraweakly continuous, completely isometric isomorphism from $H^{\infty}(E)$ onto the commutant of $\iota^{\mathcal{F}\left(E^{\sigma}\right)}\left(H^{\infty}\left(E^{\sigma}\right)\right)$. 
There is a formula for $U^{-1}$, but it is somewhat involved, as may be seen from the proof of [11, Corollary 3.10] and one of our goals is to circumvent it in calculations. Consequently, we shall not develop it here.

The thrust of Proposition 5.1 of [1] is that one can also express $\widehat{X}\left(\eta^{*}\right)$ in terms of the map defined by equation (10) and a "Cauchy" kernel expressed in terms of $\eta$ that we define as follows. Write $\eta^{(n)}: H \rightarrow E^{\otimes n} \otimes_{\sigma} H$ for the operator given by the formula:

$$
\eta^{(n)}=\left(I_{E \otimes(n-1)} \otimes \eta\right)\left(I_{E \otimes(n-2)} \otimes \eta\right) \cdots\left(I_{E} \otimes \eta\right) \eta,
$$

and write $\eta^{(0)}=I_{H}$. Clearly we have the recursive relation:

$$
\eta^{(n+1)}=\left(I_{E} \otimes \eta^{(n)}\right) \eta=\left(I_{E^{\otimes n}} \otimes \eta\right) \eta^{(n)},
$$

which is a consequence of the formulas first proved in [7, Lemmas 2.1 and 2.2].

Definition 6 The Cauchy kernel defined by an element $\eta \in \overline{\mathbb{D}\left(E^{\sigma}\right)}, C(\eta)$, is the operator from $H$ to $\mathcal{F}(E) \otimes_{\sigma} H$ given by the equation

$$
C(\eta):=\left[\begin{array}{llll}
\eta^{(0)}, & \eta^{(1)}, & \eta^{(2)}, & \cdots
\end{array}\right]^{\top} .
$$

Observe that when the norm of $\eta$ is less than $1, C(\eta)$ is bounded with norm at most $\frac{1}{1-\|\eta\|}$. For $\eta$ of norm $1, C(\eta)$ may not make sense as a bounded operator on $H$. Indeed, it is possible that $C(\eta) h$ makes sense only when $h=0$. Observe, too, that from the definition of $\eta^{(n)}$ in (11) and equation (77), we see immediately that $C(\eta)$ is an element of $\mathcal{F}(E)^{\sigma}$ when $\eta \in \mathbb{D}\left(E^{\sigma}\right)$. The following proposition is a restatement of Proposition 5.1 of [11. It is the starting point of our analysis.

Proposition 7 Let $\sigma: M \rightarrow B(H)$ be a normal representation of $M$ on a Hilbert space $H$ and let $\eta \in \mathbb{D}(E)$ be given. Further, let $\rho$ be the representation of $H^{\infty}(E)$ on $\mathcal{F}\left(E^{\sigma}\right) \otimes_{\iota} H$ defined by equation (10) and let $\iota_{H}$ be the embedding of $H$ in $\mathcal{F}\left(E^{\sigma}\right) \otimes_{\iota} H$ as the zeroth summand. Then

$$
\widehat{X}\left(\eta^{*}\right)=C(\eta)^{*} U \rho(X) \iota_{H} .
$$

\section{The Poisson Kernel}

We continue with the notation established above and let $E$ be a fixed $W^{*}$ correspondence over a von Neumann algebra $M$ and we let $\sigma$ be a normal representation on a Hilbert space $H$.

Definition 8 For $\eta$ in the closed disc $\overline{\mathbb{D}\left(E^{\sigma}\right)}$, we write $\Delta_{*}(\eta):=\left(I_{H}-\eta^{*} \eta\right)^{\frac{1}{2}}$ and we define the Poisson kernel, $K(\eta)$, by the formula,

$$
K(\eta)=\left(I_{\mathcal{F}(E)} \otimes \Delta_{*}(\eta)\right) C(\eta)=\left(I_{\mathcal{F}(E)} \otimes \Delta_{*}(\eta)\right)\left[\begin{array}{lll}
\eta^{(0)}, & \eta^{(1)}, \quad \eta^{(2)}, \cdots
\end{array}\right]^{\top},
$$

mapping $H$ to $\mathcal{F}(E) \otimes_{\sigma} H$. 
Remark 9 We note first that while $C(\eta)$ does not in general make sense as a bounded operator for $\eta$ 's with norm one, we shall see in a minute that $K(\eta)$ does. We note, too, that $\Delta_{*}(\eta)$ commutes with $\sigma(M)$ and so $I_{\mathcal{F}(E)} \otimes \Delta_{*}(\eta)$ commutes with $\sigma^{\mathcal{F}(E)}\left(H^{\infty}(E)\right)=\left\{X \otimes I_{H} \mid X \in H^{\infty}(E)\right\}$. Consequently, like the Cauchy kernel, $C(\eta)$, the Poisson kernel $K(\eta)$ lies in $\mathcal{F}(E)^{\sigma}$. It will be useful to recall that $\mathcal{F}(E)^{\sigma}$ is a $W^{*}$-correspondence over $\sigma(M)^{\prime}$. Since the action of $\sigma(M)^{\prime}$ on $H$ is given by the identity representation $\iota$, we shall denote the left action of $\sigma(M)^{\prime}$ on $\mathcal{F}(E)^{\sigma}$ by $\varphi_{\infty, \iota}$ to distinguish it from $\varphi_{\infty}$. Likewise, we write $\varphi_{\iota}$ and $\varphi_{k, \iota}$ to distinguish between the representations induced from $\sigma$ and those induced from $\iota$. So for $c \in \sigma(M)^{\prime}$ and $\eta \in \mathcal{F}(E)^{\sigma}, \varphi_{\infty, \iota}(c) \eta=\left(I_{\mathcal{F}(E)} \otimes c\right) \eta$. In particular, we may write $K(\eta)=\varphi_{\infty, \iota}\left(\Delta_{*}(\eta)\right) C(\eta)$. The inner product on $\mathcal{F}(E)^{\sigma}$ is simply $\langle X, Y\rangle=X^{*} Y$. So, for $a \in \sigma(M)^{\prime}$ and $\eta, \zeta \in \mathbb{D}\left(E^{\sigma}\right)$,

$$
\left\langle C(\eta), \varphi_{\infty, \iota}(a) C(\zeta)\right\rangle=\sum_{k}\left\langle\eta^{\otimes k}, \varphi_{k, \iota}(a) \zeta^{\otimes k}\right\rangle=\sum_{k} \theta_{\eta, \zeta}^{k}(a)=\left(i d-\theta_{\eta, \zeta}\right)^{-1}(a)
$$

where $\theta_{\eta, \zeta}(a)=\left\langle\eta, \varphi_{\iota}(a) \zeta\right\rangle$.

Proposition 10 For all $\eta \in \overline{\mathbb{D}\left(E^{\sigma}\right)}, K(\eta)$ is a contraction mapping $H$ to $\mathcal{F}(E) \otimes_{\sigma} H$. If $\|\eta\|<1$, then $K(\eta)$ is an isometry.

\section{Proof.}

$$
\begin{aligned}
& K(\eta)^{*} K(\eta)=C(\eta)^{*}\left(I_{\mathcal{F}(E)} \otimes(\Delta(\eta))^{2}\right) C(\eta) \\
& =\left[\begin{array}{llll}
\eta^{(0) *}, & \eta^{*}, & \eta^{(2) *}, & \cdots
\end{array}\right]\left(I_{\mathcal{F}(E)} \otimes(\Delta(\eta))^{2}\right)\left[\begin{array}{llll}
\eta^{(0)}, & \eta, & \eta^{(2)}, & \cdots
\end{array}\right]^{T} \\
& =\sum_{n} \eta^{(n) *}\left(I_{E^{\otimes n}} \otimes \Delta(\eta)\right)^{2} \eta^{(n)} \\
& =\lim _{N \rightarrow \infty} \sum_{n=0}^{N} \eta^{(n) *} \eta^{(n)}-\eta^{(n+1) *} \eta^{(n+1)}=I_{H}-\lim _{N \rightarrow \infty} \eta^{(N+1) *} \eta^{(N+1)} .
\end{aligned}
$$

The passage from the third line to the fourth is a consequence of equation 12. Also, by 12 and the fact that the norm of $\eta$ is at most one, the sequence $\left\{\eta^{(N+1) *} \eta^{(N+1)}\right\}_{N \geq 0}$ is a decreasing sequence of contractions on $H$. Therefore the sequence $\left\{I_{H}-\eta^{(N+1) *} \eta^{(N+1)}\right\}$ converges strongly to a contraction on $H$. The limit is $I_{H}$ if $\eta$ is a strict contraction.

The following lemma shows that the values of the Poisson kernel are "operator eigenvectors" for the adjoints of the creation operators. The "operator eigenvalue" for $T_{\xi}^{*} \otimes I$ determined by $\eta \in \mathbb{D}\left(E^{\sigma}\right)$ is $\widehat{\eta}^{*}(\xi)^{*}$.

Lemma 11 For all $\xi \in E$ and all $\eta \in \mathbb{D}\left(E^{\sigma}\right)$,

$$
\left(T_{\xi}^{*} \otimes I\right) K(\eta)=K(\eta) \hat{\eta}^{*}(\xi)^{*} .
$$

Proof. Since $\|\eta\|<1$, the operator on $H, \Delta_{*}(\eta)$, is invertible. Also, $I \otimes \Delta_{*}(\eta)$ commutes with $\left(T_{\xi} \otimes I\right)^{*}$ so it suffices to prove that $\widehat{\eta^{*}}(\xi) C(\eta)^{*}=C(\eta)^{*}\left(T_{\xi} \otimes I\right)$ 
as operators from $\mathcal{F}(E) \otimes_{\sigma} H$ to $H$. To prove equality, it suffices to evaluate both sides on an element of the form $\zeta \otimes h \in E^{\otimes n} \otimes H$. By definition of $C(\eta)$ and the formula (11),

$$
\begin{aligned}
C(\eta)^{*}(\zeta \otimes h) & =\eta^{(n) *}(\zeta \otimes h) \\
& =\eta^{*}\left(I_{E} \otimes \eta\right)^{*} \cdots\left(I_{E^{\otimes(n-1)}} \otimes \eta\right)^{*}(\zeta \otimes h) .
\end{aligned}
$$

Consequently,

$$
\begin{aligned}
& \widehat{\eta^{*}}(\xi) C(\eta)^{*}(\zeta \otimes h)=\widehat{\eta^{*}}(\xi) \eta^{*}\left(I_{E} \otimes \eta\right)^{*} \cdots\left(I_{E^{\otimes(n-1)}} \otimes \eta\right)^{*}(\zeta \otimes h) \\
& =\eta^{*}\left(\xi \otimes\left(\eta^{*}\left(I_{E} \otimes \eta\right)^{*} \cdots\left(I_{E \otimes(n-1)} \otimes \eta\right)^{*}(\zeta \otimes h)\right)\right. \\
& =\eta^{*}\left(I_{E} \otimes \eta\right)^{*} \cdots\left(I_{E \otimes(n-1)} \otimes \eta\right)^{*}\left(I_{E \otimes n} \otimes \eta\right)^{*}(\xi \otimes \zeta \otimes h) \\
& =C(\eta)^{*}(\xi \otimes \eta \otimes h) \\
& =C(\eta)^{*}\left(T_{\xi} \otimes I\right)(\zeta \otimes h) \text {. }
\end{aligned}
$$

Theorem 12 For all $\eta \in \mathbb{D}\left(E^{\sigma}\right)$ and all $X \in H^{\infty}(E)$,

$$
K(\eta) \widehat{X}\left(\eta^{*}\right)^{*}=\left(X^{*} \otimes I_{H}\right) K(\eta)
$$

and

$$
\widehat{X}\left(\eta^{*}\right)=K(\eta)^{*}(X \otimes I) K(\eta) .
$$

Proof. Remark 9 and Proposition 11 show that formula (14) holds for all $X$ of the form $X=T_{\xi}$ and $X=\varphi_{\infty}(a), \xi \in E$ and $a \in M$. (Note that $\left.\hat{\eta}^{*}(\xi)=\hat{T}_{\xi}\left(\eta^{*}\right)\right)$. Further these two results show that the range of $K(\eta)$ is invariant under all these operators. Thus the formula holds for the ultraweakly closed algebra of operators generated by all the $T_{\xi}$ and all the $\varphi_{\infty}(a), \xi \in E$ and $a \in M$. Thus the formula (14) holds for all $X \in H^{\infty}(E)$. See the discussion on page 384 of [11] and [11, Corollary 2.14]. Equation (15) follows from (14) since $K(\eta)$ is an isometry.

Remark 13 Formula 15 gives another proof that the minimal isometric dilation of the representation of $H^{\infty}(E)$ on $H$ determined by $\eta$ in the open disc $\mathbb{D}\left(E^{\sigma}\right)$ is an induced representation of $H^{\infty}(E)$ acting on $\mathcal{F}(E) \otimes_{\sigma} H: X \rightarrow X \otimes I$ [11, Theorem 2.13].

The following theorem is our replacement for [11, Proposition 5.1]. It captures more clearly the roles played by the various constructs. We let $\iota_{H}$ denote the embedding of $H$ into $\mathcal{F}(E) \otimes_{\sigma} H$, and we write $P_{H}$ for its adjoint. Also, $\rho$ is the representation of $H^{\infty}\left(E^{\sigma}\right)$ defined in equation (9).

Theorem 14 For all $\eta \in \mathbb{D}\left(E^{\sigma}\right)$ and all $X \in H^{\infty}(E), K(\eta)=\rho(\Delta(\eta)(I-$ $\left.\left.T_{\eta}\right)^{-1}\right) \iota_{H}$, and

$$
\begin{aligned}
\hat{X}\left(\eta^{*}\right) & =K(\eta)^{*}(X \otimes I) K(\eta) \\
& \left.\left.=P_{H} \rho\left(\Delta_{*}(\eta)\left(I-T_{\eta}\right)^{-1}\right)\right)^{*}\left(X \otimes I_{H}\right) \rho\left(\Delta_{*}(\eta)\left(I-T_{\eta}\right)^{-1}\right)\right) \iota_{H} \\
& \left.=P_{H}\left\{\rho\left(\left(I-T_{\eta}\right)^{-1}\right)^{*}\left(I_{\mathcal{F}(E)} \otimes \Delta_{*}(\eta)^{2}\right) \rho\left(\left(I-T_{\eta}\right)^{-1}\right)\right)\right\}\left(X \otimes I_{H}\right) \iota_{H} .
\end{aligned}
$$


Proof. Since $I_{\mathcal{F}(E)} \otimes \Delta_{*}(\eta)=\rho\left(\Delta_{*}(\eta)\right)$ by [11, Theorem 3.9], it suffices to prove that $C(\eta)=\rho\left(\left(I-T_{\eta}\right)^{-1}\right) \iota_{H}$. Since $\left(I-T_{\eta}\right)^{-1}=\sum_{n=0}^{\infty} T_{\eta}^{n}$, it suffices to note that for $h \in H, \rho\left(T_{\eta}^{n}\right) h=U(\eta \otimes \eta \cdots \otimes \eta \otimes h)=\left(I_{E \otimes(n-1)} \otimes \eta\right)\left(I_{E \otimes(n-2)} \otimes\right.$ $\eta) \cdots\left(I_{E} \otimes \eta\right) \eta h=\eta^{(n)} h$.

\section{Characteristic Operator Functions and Canon- ical Models}

In [12] we studied canonical models for representations of the Hardy algebras. So, given $\eta \in \mathbb{D}\left(E^{\sigma}\right)$, it makes sense and is of interest to investigate how the canonical model of the representation $\sigma \times \widehat{\eta^{*}}$ is related to the Poisson kernel $K(\cdot)$. We shall see that they are closely related. We fix $\eta \in \mathbb{D}\left(E^{\sigma}\right)$ for the rest of this section and in the computations that follow, we write $\Delta_{*}=\Delta_{*}(\eta)$, which recall is $\left(I_{H}-\eta^{*} \eta\right)^{1 / 2}$, and we write $\Delta=\Delta(\eta):=\left(I_{E \otimes H}-\eta \eta^{*}\right)^{1 / 2}$ for the defect operators associated with $\eta^{*}$. Note that since $\eta$ has norm strictly less than one, the operators $\Delta$ and $\Delta_{*}$ are invertible. Therefore their ranges are all of $E \otimes H$ and $H$, respectively. Nevertheless, to be consistent with the literature, we continue to denote the range of $\Delta$ by $\mathcal{D}$ and the range of $\Delta_{*}$ by $\mathcal{D}_{*}$. We already have noted that $\Delta_{*}$ commutes with $\sigma(M)$ and it is immediate that $\Delta$ commutes with $\varphi(M) \otimes I_{H}$. The characteristic operator of $\widehat{\eta^{*}}$ (or, of $\left.\left(\widehat{\eta}^{*}, \sigma\right)\right)$ is defined in 12, Equation (12)] to be an operator $\Theta_{\widehat{\eta^{*}}}: \mathcal{F}(E) \otimes_{\rho} \mathcal{D} \rightarrow \mathcal{F}(E) \otimes_{\rho} \mathcal{D}_{*}$ whose complete development need not be rehearsed here (in particular, the subscript $\rho$ in the notation need not concern us). We will give a different definition whose equivalence with the one in 12, will follow easily from the next lemma. It will have the advantage that it leads immediately to a matrix representation that is useful for our purposes. To simplify notation, we shall write $\Theta_{\eta}$ for $\Theta_{\widehat{\eta^{*}}}$.

Lemma 15 For $i=1,2$ let $\sigma_{i}$ be a faithful normal representation of $M$ on the Hilbert space $\mathcal{E}_{i}$ and let $Y$ be a bounded linear transformation mapping $\mathcal{F}(E) \otimes_{\sigma_{1}}$ $\mathcal{E}_{1} \rightarrow \mathcal{F}(E) \otimes_{\sigma_{2}} \mathcal{E}_{2}$. If $Y$ intertwines $\sigma_{1}^{\mathcal{F}(E)}$ and $\sigma_{2}^{\mathcal{F}(E)}$, then $Y$ is completely determined by its values on $\mathcal{E}_{1}$. Conversely, given an operator $Y_{0}$ from $\mathcal{E}_{1}$ to $\mathcal{F}(E) \otimes_{\sigma_{2}} \mathcal{E}_{2}$, the formula

$$
Y(\xi \otimes e)=\xi \otimes Y_{0} e,
$$

$\xi \otimes e \in \mathcal{F}(E) \otimes_{\sigma_{1}} \mathcal{E}_{1}$ defines a bounded operator $Y: \mathcal{F}(E) \otimes_{\sigma_{1}} \mathcal{E}_{1} \rightarrow \mathcal{F}(E) \otimes_{\sigma_{2}} \mathcal{E}_{2}$ that intertwines $\sigma_{1}^{\mathcal{F}(E)}$ and $\sigma_{2}^{\mathcal{F}(E)}$.

Proof. The proof is immediate from Theorem 5. The only thing that might be at issue is how to handle different spaces and different representations of $M$, $\left(\sigma_{i}, \mathcal{E}_{i}\right), i=1,2$. One simply forms the direct sum of $\sigma_{1}$ and $\sigma_{2}$ and induces that. Operators on the resulting space $\mathcal{F}(E) \otimes\left(\mathcal{E}_{1} \oplus \mathcal{E}_{2}\right)=\mathcal{F}(E) \otimes\left(\mathcal{E}_{1}\right) \oplus \mathcal{F}(E) \otimes\left(\mathcal{E}_{2}\right)$ have a $2 \times 2$ matrix representation, and operators that intertwine $\sigma_{1}^{\mathcal{F}(E)}$ and $\sigma_{2}^{\mathcal{F}(E)}$ can be realized as matrices of the form $\left(\begin{array}{cc}0 & 0 \\ Y & 0\end{array}\right)$. 
To define the characteristic operator, $\Theta_{\eta}$, determined by an element $\eta \in$ $\mathbb{D}\left(E^{\sigma}\right)$, we note that the analysis found in [12, pp. 429-430] shows that the operator $\theta_{\eta}$ defined on $\mathcal{D}$ by the formula,

$$
\theta_{\eta} d=-\eta^{*} d+\left(I_{1} \otimes \Delta_{*}\right) \Delta d+\sum_{k=2}^{\infty}\left(I_{k} \otimes \Delta_{*}\right)\left(I_{1} \otimes \eta^{(k-2)}\right) \Delta d
$$

for $d \in \mathcal{D}$, is a bounded linear operator from $\mathcal{D}$ to $\mathcal{F}(E) \otimes_{\sigma} \mathcal{D}_{*}$.

Definition 16 For $\eta \in E^{\sigma}$, the characteristic operator determined by $\eta$ is the operator $\Theta_{\eta}: \mathcal{F}(E) \otimes_{\varphi \otimes I \mid \mathcal{D}} \mathcal{D} \rightarrow \mathcal{F}(E) \otimes_{\sigma} \mathcal{D}_{*}$ defined by the formula

$$
\Theta_{\eta}(\xi \otimes d)=\xi \otimes \theta_{\eta} d
$$

for $d \in D$ and $\xi \in \mathcal{F}(E)$.

Our next objective is to prove the following theorem which is the principal result of this section. It was inspired in part by Popescu's analysis in [16] and [17. See [17, Theorem 3.2].

Theorem 17 For $\eta \in \mathbb{D}\left(E^{\sigma}\right)$, the Poisson kernel $K(\eta)$ and the characteristic operator $\Theta_{\eta}$ are related by the equation

$$
I=K(\eta) K(\eta)^{*}+\Theta_{\eta} \Theta_{\eta}^{*}
$$

on $\mathcal{F}(E) \otimes_{\sigma} \mathcal{D}_{*}$.

Proof. With respect to the decompositions $\mathcal{F}(E) \otimes \mathcal{D}=\mathcal{D} \oplus E \otimes \mathcal{D} \oplus E^{\otimes 2} \otimes \mathcal{D} \oplus \ldots$ and $\mathcal{F}(E) \otimes \mathcal{D}_{*}=\mathcal{D}_{*} \oplus E \otimes \mathcal{D}_{*} \oplus E^{\otimes 2} \otimes \mathcal{D}_{*} \oplus \ldots, \Theta_{\eta}$ can be written in a matricial form $\Theta_{\eta}=\left(\Theta_{i, j}\right)_{i, j=0}^{\infty}$ where $\Theta_{i, j}: E^{\otimes j} \otimes \mathcal{D} \rightarrow E^{\otimes i} \otimes \mathcal{D}_{*}$. It follows from (17) that, for $i<j, \Theta_{i, j}=0$. For $i=j$, we have $\Theta_{j, j}=I_{j} \otimes\left(-\eta^{*}\right)$ and, for $i>j, \Theta_{i, j}=I_{j} \otimes\left(I_{i-j} \otimes \Delta_{*}\right)\left(I_{1} \otimes \eta^{(i-j-1)}\right) \Delta \mid \mathcal{D}$. This enables us to write the matricial form of $\Theta_{\eta} \Theta_{\eta}^{*}$ (with respect to the decomposition $\mathcal{F}(E) \otimes \mathcal{D}_{*}=$ $\left.\mathcal{D}_{*} \oplus E \otimes \mathcal{D}_{*} \oplus E^{\otimes 2} \otimes \mathcal{D}_{*} \oplus \ldots\right)$. We start with the diagonal entries.

$$
\begin{gathered}
\left(\Theta_{\eta} \Theta_{\eta}^{*}\right)_{k, k}=\sum_{l=0}^{k} \Theta_{k, l} \Theta_{k, l}^{*}= \\
I_{k} \otimes \eta^{*} \eta+\sum_{m=1}^{k} I_{k-m} \otimes\left(I_{m} \otimes \Delta_{*}\right)\left(I_{1} \otimes \eta^{(m-1)}\right) \Delta^{2}\left(I_{1} \otimes \eta^{(m-1) *}\right)\left(I_{m} \otimes \Delta_{*}\right) .
\end{gathered}
$$

$\operatorname{But}\left(I_{1} \otimes \eta^{(m-1)}\right) \Delta^{2}\left(I_{1} \otimes \eta^{(m-1) *}\right)=\left(I_{1} \otimes \eta^{(m-1)}\right)\left(I_{E \otimes H}-\eta \eta^{*}\right)\left(I_{1} \otimes \eta^{(m-1) *}\right)=$ $I_{1} \otimes \eta^{(m-1)} \eta^{(m-1) *}-\eta^{(m)} \eta^{(m) *}$ and we get

$$
\left(\Theta_{\eta} \Theta_{\eta}^{*}\right)_{k, k}=I_{k} \otimes \eta^{*} \eta+\sum_{m=1}^{k} I_{k-m+1} \otimes\left(I_{m-1} \otimes \Delta_{*}\right) \eta^{(m-1)} \eta^{(m-1) *}\left(I_{m-1} \otimes \Delta_{*}\right)-
$$




$$
\begin{gathered}
\sum_{m=1}^{k} I_{k-m} \otimes\left(I_{m} \otimes \Delta_{*}\right) \eta^{(m)} \eta^{(m) *}\left(I_{m} \otimes \Delta_{*}\right)=I_{k} \otimes \eta^{*} \eta+I_{k} \otimes \Delta_{*}^{2}- \\
\left(I_{k} \otimes \Delta_{*}\right) \eta^{(k)} \eta^{(k) *}\left(I_{k} \otimes \Delta_{*}\right)=I_{E^{\otimes k} \otimes H}-\left(I_{k} \otimes \Delta_{*}\right) \eta^{(k)} \eta^{(k) *}\left(I_{k} \otimes \Delta_{*}\right) .
\end{gathered}
$$

Now, fix $l<k$. Then $\left(\Theta_{\eta} \Theta_{\eta}^{*}\right)_{k, l}=\sum_{m=0}^{l} \Theta_{k, m} \Theta_{l, m}^{*}$. When $m=l$ we get

$$
\begin{gathered}
\Theta_{k, l} \Theta_{l, l}^{*}=I_{l} \otimes\left(I_{k-l} \otimes \Delta_{*}\right)\left(I_{1} \otimes \eta^{(k-l-1)}\right) \Delta(-\eta)= \\
-I_{l} \otimes\left(I_{k-l} \otimes \Delta_{*}\right)\left(I_{1} \otimes \eta^{(k-l-1)}\right) \eta \Delta_{*}=-I_{l} \otimes\left(I_{k-l} \otimes \Delta_{*}\right) \eta^{(k-l)} \Delta_{*} .
\end{gathered}
$$

For $m<l, \Theta_{k, m} \Theta_{l, m}^{*}=$

$$
I_{m} \otimes\left(I_{k-m} \otimes \Delta_{*}\right)\left(I_{1} \otimes \eta^{(k-m-1)}\right) \Delta^{2}\left(I_{1} \otimes \eta^{(l-m-1) *}\right)\left(I_{l-m} \otimes \Delta_{*}\right) .
$$

$\operatorname{But}\left(I_{1} \otimes \eta^{(k-m-1)}\right) \Delta^{2}\left(I_{1} \otimes \eta^{(l-m-1) *}\right)=I_{1} \otimes \eta^{(k-m-1)} \eta^{(l-m-1) *}-\eta^{(k-m)} \eta^{(l-m) *}$. Hence

$$
\begin{gathered}
\Theta_{k, m} \Theta_{l, m}^{*}=I_{m} \otimes\left(I_{k-m} \otimes \Delta_{*}\right)\left(I_{1} \otimes \eta^{(k-m-1)} \eta^{(l-m-1) *}\right)\left(I_{l-m} \otimes \Delta_{*}\right)- \\
I_{m} \otimes\left(I_{k-m} \otimes \Delta_{*}\right)\left(I_{1} \otimes \eta^{(k-m)} \eta^{(l-m-) *}\right)\left(I_{l-m} \otimes \Delta_{*}\right) .
\end{gathered}
$$

Thus

$$
\begin{gathered}
\left(\Theta_{\eta} \Theta_{\eta}^{*}\right)_{k, l}=-I_{l} \otimes\left(I_{k-l} \otimes \Delta_{*}\right) \eta^{(k-l)} \Delta_{*}+I_{l-1} \otimes\left(I_{k-l+1} \otimes \Delta_{*}\right)\left(I_{1} \otimes \eta^{(k-l)}\right)\left(I_{1} \otimes \Delta_{*}\right)- \\
\left(I_{k} \otimes \Delta_{*}\right) \eta^{(k)} \eta^{(l) *}\left(I_{l} \otimes \Delta_{*}\right)=-\left(I_{k} \otimes \Delta_{*}\right) \eta^{(k)} \eta^{(l) *}\left(I_{l} \otimes \Delta_{*}\right) .
\end{gathered}
$$

It is easy to check, using the definition of $K(\eta)$, that the matricial form of $K(\eta) K(\eta)^{*}$ is

$$
\left(K(\eta) K(\eta)^{*}\right)_{k, l}=\left(I_{k} \otimes \Delta_{*}\right) \eta^{(k)} \eta^{(l) *}\left(I_{l} \otimes \Delta_{*}\right)
$$

and we conclude

$$
\Theta_{\eta} \Theta_{\eta}^{*}+K(\eta) K(\eta)^{*}=I
$$

\section{Point Evaluations on $\mathbb{D}(E)$}

Recall from [11, Theorem 3.6] that there is a natural isomorphism between $E$ and $\left(E^{\sigma}\right)^{\iota}$, where $\iota$ denotes the identity representation of $\sigma(M)^{\prime}$ on $H$. Thus we may identify $E$ and $\left(E^{\sigma}\right)^{\iota}$ and view elements of $H^{\infty}\left(E^{\sigma}\right)$ as functions on $\mathbb{D}(E)$. This will help to shed some light on the relation between our work and [1] and it will enable us to (anti)represent $H^{\infty}(E)$ in the algebra of completely bounded maps on $M, C B(M)$. For this purpose, we adopt the convention that when $X \in H^{\infty}(E)$ and when we write $X 1,1$ is understood to be the identity of $M$ viewed as a vector of $\mathcal{F}(E)=M \oplus E \oplus \ldots$. So $X 1 \in \mathcal{F}(E)$. We write 
$C(\xi)$ and $K(\xi)$, for $\xi \in \mathbb{D}(E)$, using the obvious modifications of Definitions 6 and 8 and note that $K(\xi)=\varphi_{\infty}\left(\Delta_{*}\right) C(\xi)$ where $\Delta_{*}=(I-\langle\xi, \xi\rangle)^{1 / 2}$. Also, we write $\mathbb{E}_{0}$ for the conditional expectation of $H^{\infty}(E)$. This map is defined as $\Phi_{0}$ on page 336 of [11. It picks off the zeroth coefficient of an element $X \in H^{\infty}(E)$ calculated with respect to the gauge automorphism group.

Theorem 18 For $\xi \in \mathbb{D}(E)$, and $X \in H^{\infty}(E)$, we define the map $\Phi_{X}^{\xi}: M \rightarrow$ $M$ by the formula

$$
\Phi_{X}^{\xi}(a)=\left\langle C(\xi), \varphi_{\infty}(a) X 1\right\rangle
$$

for all $a \in M$. Then

(1) For each $a \in M, \Phi_{X}^{\xi}(a)$ is the unique element of $M$ such that

$$
\left(I-T_{\xi}^{*}\right)^{-1}\left(\varphi_{\infty}(a) X-\varphi_{\infty}\left(\Phi_{X}^{\xi}(a)\right)\right) \in H_{0}^{\infty}(E),
$$

where $H_{0}^{\infty}(E):=\bigvee\left\{T_{\xi} X \mid \xi \in E, X \in H^{\infty}(E)\right\}=H^{\infty}(E) \cap \operatorname{Ker}\left(\mathbb{E}_{0}\right)$.

(2) We have

$$
X^{*} \varphi_{\infty}(a) C(\xi)=\varphi_{\infty}\left(\Phi_{X}^{\xi}(a)^{*}\right) C(\xi)
$$

and, in particular,

$$
X^{*} K(\xi)=\varphi_{\infty}\left(\Phi_{X}^{\xi}\left(\Delta_{*}\right)^{*} \Delta_{*}^{-1}\right) K(\xi) .
$$

So that $K(\xi)$ is an eigenvector of $X^{*}$ (cf. Corollary [12).

(3) For each $\xi \in \mathbb{D}(E)$, the map $\Phi: X \mapsto \Phi_{X}^{\xi}$ is an algebra antihomomorphism from $H^{\infty}(E)$ into $C B(M)$.

Proof. First note that, since $\|\xi\|<1, I-T_{\xi}^{*}$ is an invertible operator on $\mathcal{F}(E)$ with inverse equal to $I+T_{\xi}^{*}+T_{\xi}^{* 2}+\ldots$ We claim that for $X \in H^{\infty}(E)$, $\left(I+T_{\xi}^{*}+T_{\xi}^{* 2}+\ldots\right)\left(\varphi_{\infty}(a) X-\varphi_{\infty}\left(\Phi_{X}^{\xi}(a)\right)\right)$ lies in $H_{0}^{\infty}(E)$. If $X=T_{g}$ for some $g \in E^{\otimes n}$, then

$$
\begin{gathered}
\left(I+T_{\xi}^{*}+T_{\xi}^{* 2}+\ldots\right)\left(\varphi_{\infty}(a) X-\varphi_{\infty}\left(\Phi_{X}^{\xi}(a)\right)\right)= \\
\left(I+T_{\xi}^{*}+T_{\xi}^{* 2}+\ldots\right)\left(\varphi_{\infty}(a) T_{g}-\varphi_{\infty}\left(\left\langle\xi^{\otimes n}, \varphi_{n}(a) g\right\rangle\right)\right) .
\end{gathered}
$$

Note, too, that $T_{\xi}^{* k} \varphi_{\infty}(a) T_{g}=T_{\xi}^{*(k-n)} \varphi_{\infty}\left(\left\langle\xi^{\otimes n}, \varphi_{n}(a) g\right\rangle\right)$, for $k \geq n$. Thus

$$
\begin{aligned}
& \left(I+T_{\xi}^{*}+T_{\xi}^{* 2}+\ldots\right)\left(\varphi_{\infty}(a) X-\varphi_{\infty}\left(\Phi_{X}^{\xi}(a)\right)\right) \\
= & \varphi_{\infty}(a) T_{g}+T_{\xi}^{*} \varphi_{\infty}(a) T_{g}+T_{\xi}^{* 2} \varphi_{\infty}(a) T_{g}+\ldots+T_{\xi}^{*(n-1)} \varphi_{\infty}(a) T_{g} \in H_{0}^{\infty}(E) .
\end{aligned}
$$

It follows that the result holds for all operators in a ultraweakly-dense subalgebra of $H^{\infty}(E)$. Since the map taking $X \in H^{\infty}(E)$ to $\Phi_{X}^{\xi}(a)$ is ultraweaklycontinuous, $\left(I+T_{\xi}^{*}+T_{\xi}^{* 2}+\ldots\right)\left(\varphi_{\infty}(a) X-\varphi_{\infty}\left(\Phi_{X}^{\xi}(a)\right)\right)$ lies in $H_{0}^{\infty}(E)$ for all 
$X \in H^{\infty}(E)$. To prove uniqueness we need to show that, if $c \in \varphi_{\infty}(M)$ satisfies $\left(I-T_{\xi}^{*}\right)^{-1} c \in H^{\infty}(E)_{0}$, then $c=0$. But, since $\left(I-T_{\xi}^{*}\right)^{-1} c=\left(I+T_{\xi}^{*}+T_{\xi}^{* 2}+\ldots\right) c$, this is clear and (1) follows.

To prove (2), fix $X \in H^{\infty}(E), a \in M$ and write $Y$ for $\left(I-T_{\xi}^{*}\right)^{-1}\left(\varphi_{\infty}(a) X-\right.$ $\left.\varphi_{\infty}\left(\Phi_{X}^{\xi}(a)\right)\right)$ (in $H_{0}^{\infty}(E)$ ). Then $\left(\varphi_{\infty}(a) X-\varphi_{\infty}\left(\Phi_{X}^{\xi}(a)\right)\right)^{*}=Y^{*}\left(I-T_{\xi}\right)$. Since $\left(I-T_{\xi}\right) C(\xi)=1 \in \mathcal{F}(E)$, and $Y \in H_{0}^{\infty}(E)$, we have $\left(\varphi_{\infty}(a) X-\right.$ $\left.\varphi_{\infty}\left(\Phi_{X}^{\xi}(a)\right)\right)^{*} C(\xi)=Y^{*}\left(I-T_{\xi}\right) C(\xi)=0$. This, together with the observation that $K(\xi)=\varphi_{\infty}\left(\Delta_{*}\right) C(\xi)$, completes the proof of (2).

Finally, note that the linearity of the map $X \rightarrow \Phi_{X}^{\xi}$ is obvious and antimultiplicativity follows from the computation $\Phi_{X Z}^{\xi}(a)=\left\langle X^{*} \varphi_{\infty}\left(a^{*}\right) C(\xi), Z 1\right\rangle=$ $\left\langle\varphi_{\infty}\left(\Phi_{X}^{\xi}(a)^{*}\right) C(\xi), Z 1\right\rangle=\Phi_{Z}^{\xi}\left(\Phi_{X}^{\xi}(a)\right)$.

Remark 19 (i) When we fix $X$ and $\xi$ and let $a=I \in M$, we find that $\Phi_{X}^{\xi}(I)$ is very closely related to the concept of "left point evaluation" of $X$ at $\xi$ that was defined for the special case of upper triangular operators in [1] and studied there and in subsequent papers by various authors. (Compare [1], Theorem 3.3] with Theorem 18(1)). If one adopts the "reproducing kernel correspondence" point of view discussed in Remark 9, this indeed can be viewed as a point evaluation. Note, however, that the map $X \mapsto \Phi_{X}^{\xi}(I)$ is not multiplicative in general. (See also [13, Example 2.25]).

(ii) It follows from Theorem 18 that, for each $\xi \in \mathbb{D}(E)$, the kernel of the map $X \rightarrow \Phi_{X}^{\xi}$ is a two-sided ideal in $H^{\infty}(E)$.

\section{Curvature}

In this section we express the curvature invariant that we attached to completely positive maps on semifinite factors [9] in terms of the Poisson kernel. This provides a further connection between that work and the analysis by Popescu in [16] and the study by Arveson [2]. We suppose from now on that $M$ is a semifinite factor and we fix a faithful normal semifinite trace $\tau$ on $M$. We recall that once $\tau$ is fixed, we may define a dimension for any representation and we can assign a natural trace to the commutant of the representation (cf. [9, Definition 2.1]). Specifically, if $\sigma$ is a normal representation of $M$ on $H$, then there is a Hilbert space isometry $u$ from $H$ to $L^{2}(M, \tau) \otimes \ell^{2}(\mathbb{N})$, where $L^{2}(M, \tau)$ is the $L^{2}$-space canonically associated with $\tau$, i.e., the GNS-space, such that $u \sigma(a)=\lambda(a) \otimes I_{\ell^{2}(\mathbb{N})} u$, for all $a \in M$, where $\lambda$ is the left representation of $M$ on $L^{2}(M, \tau)$. The range projection of $u, e$, lies in the commutant of $\lambda(M) \otimes$ $I_{\ell^{2}(\mathbb{N})}$, which is $\rho(M) \otimes B\left(\ell^{2}(\mathbb{N})\right)$, where $\rho$ is the right (anti) representation of $M$ on $L^{2}(M, \tau)$. The usual trace on $\rho(M) \otimes B\left(\ell^{2}(\mathbb{N})\right)$ is $\tau \otimes t r$, where $t r$ is the standard trace on $B\left(\ell^{2}(\mathbb{N})\right)$, i.e., the one that assigns to each projection in $B\left(\ell^{2}(\mathbb{N})\right)$ its rank. Then, while $u$ and $e$ are not unique, the Murray-von Neumann equivalence class of $e$ in $\rho(M) \otimes B\left(\ell^{2}(\mathbb{N})\right)$ is uniquely determined by $\sigma$ and so, therefore, $\tau \otimes \operatorname{tr}(e) \in[0, \infty]$ is unique. This number is called the dimension of $H$ (or of $\sigma$ ) as a module over $M$. We write this number $\operatorname{dim}_{\sigma} H$. 
It will be important to remember, too, that the commutant of $\sigma(M)$ is spatially isomorphic to $e\left(\rho(M) \otimes B\left(\ell^{2}(\mathbb{N})\right)\right) e$ via $u$ and so we can refer to the natural trace on $\sigma(M)^{\prime}$ as the restriction of $\tau \otimes \operatorname{tr}$ to $e\left(\rho(M) \otimes B\left(\ell^{2}(\mathbb{N})\right)\right) e$. We shall do this and we shall denote it by $\operatorname{tr}_{\sigma(M)^{\prime}}$. If $E$ is a $W^{*}$-correspondence over $M$ of the kind we have been studying, then the (left) dimension of $E$ is defined to be the dimension of the representation $\varphi \otimes I$, representing $M$ on $E \otimes_{\lambda} L^{2}(M, \tau)$. We denote this dimension by $\operatorname{dim}_{l}(E)$. (See [9, Definition 2.5].)

An $\eta \in \overline{\mathbb{D}\left(E^{\sigma}\right)}$ defines a completely positive map $P=P_{\eta}$ on $\sigma(M)^{\prime}$ via the formula $P(a)=\eta^{*}\left(I_{E} \otimes a\right) \eta, a \in \sigma(M)^{\prime}$. Alternatively, given the formula for the inner product in $E^{\sigma}, P(a)=\langle\eta, a \eta\rangle$. And conversely, given a completely positive map $P$ on $\sigma(M)^{\prime}$ there a $W^{*}$-correspondence $E$ over $M$ and an $\eta \in \overline{\mathbb{D}\left(E^{\sigma}\right)}$ such that $P=P_{\eta}[8$, Corollary 2.23].

Definition 20 Let $E$ be a $W^{*}$-correspondence over the von Neumann algebra $M$ with $\operatorname{dim}_{l}(E):=d$, and let $\sigma$ be a representation of $M$ on the Hilbert space $H$. Then for $\eta \in \overline{\mathbb{D}\left(E^{\sigma}\right)}$, the curvature of $\eta$ is defined to be the curvature of $P_{\eta}$ in the sense of [9, Definition 3.1], which is the limit

$$
\lim _{N \rightarrow \infty} \frac{\operatorname{tr}_{\sigma(M)^{\prime}}\left(I-P_{\eta}^{N+1}\left(I_{H}\right)\right)}{\sum_{k=0}^{N} d^{k}}
$$

and will be denoted $\kappa(\eta)$.

The limit exists, as was shown in [9, Theorem 3.3], where alternate formulas for $\kappa(\eta)$ may also be found. The basis for the calculations we make here is the following lemma, whose proof may be assembled easily from [9].

Lemma 21 Let $E$ and $F$ be $W^{*}$-correspondences over $M$ and let $\sigma$ be normal representation of $M$ on a Hilbert space $H$. Then

1. $\operatorname{dim}_{l}(E \otimes F)=\operatorname{dim}_{l} E \times \operatorname{dim}_{l} F$.

2. If $\eta, \zeta \in E^{\sigma}$, then

$$
\operatorname{tr}_{\sigma(M)^{\prime}}\left(\zeta^{*} \eta\right)=\operatorname{tr}_{\left(\varphi(M) \otimes I_{H}\right)^{\prime}}\left(\eta \zeta^{*}\right)
$$

where $\varphi$ denotes the left action of $M$ on $E$.

3. For all positive $x$ in $\sigma(M)^{\prime}, \operatorname{tr}_{\left(\varphi(M) \otimes I_{H}\right)^{\prime}}\left(I_{E} \otimes x\right)=\operatorname{tr}_{\sigma(M)^{\prime}}(x) \cdot \operatorname{dim}_{l} E$.

4. $\operatorname{dim}_{\varphi \otimes I_{H}}\left(E \otimes_{\sigma} H\right)=\operatorname{dim}_{l}(E) \cdot \operatorname{dim}_{\sigma} H$.

Proof. The first assertion is proved as Corollary 2.8 in [9. The second assertion is embedded in the proof of [9, Proposition 2.12]. For the sake of clarity we repeat the salient part of it here. Form the direct sum $H \oplus\left(E \otimes_{\sigma} H\right)$ and let $\tilde{\sigma}=\sigma \oplus\left(\varphi \otimes I_{H}\right)$ be the representation of $M$ acting on this space. Then the commutant of $\tilde{\sigma}(M)$ is the set of all matrices of the form $\left(\begin{array}{ll}a & b \\ c & d\end{array}\right)$ where 
$a \in \sigma(M)^{\prime}, d \in\left(\varphi(M) \otimes I_{H}\right)^{\prime}, b \varphi(x) \otimes I_{H}=\sigma(x) b$ and $c \sigma(x)=\varphi(x) \otimes I_{H} c$ for all $x \in M$. Further, it is easy to see that $\operatorname{tr}_{\tilde{\sigma}(M)^{\prime}}\left(\begin{array}{ll}a & b \\ c & d\end{array}\right)=\operatorname{tr}_{\sigma(M)^{\prime}}(a)+$ $\operatorname{tr}_{\left(\varphi(M) \otimes I_{H}\right)^{\prime}}(d)$. Thus we find that

$$
\begin{aligned}
& \operatorname{tr}_{\sigma(M)^{\prime}}\left(\zeta^{*} \eta\right)=\operatorname{tr}_{\tilde{\sigma}(M)^{\prime}}\left(\begin{array}{cc}
\zeta^{*} \eta & 0 \\
0 & 0
\end{array}\right) \\
& =\operatorname{tr}_{\tilde{\sigma}(M)^{\prime}}\left(\left(\begin{array}{cc}
0 & \zeta^{*} \\
0 & 0
\end{array}\right)\left(\begin{array}{ll}
0 & 0 \\
\eta & 0
\end{array}\right)\right)=\operatorname{tr}_{\tilde{\sigma}(M)^{\prime}}\left(\left(\begin{array}{ll}
0 & 0 \\
\eta & 0
\end{array}\right)\left(\begin{array}{cc}
0 & \zeta^{*} \\
0 & 0
\end{array}\right)\right) \\
& \quad=\operatorname{tr}_{\tilde{\sigma}(M)^{\prime}}\left(\begin{array}{cc}
0 & 0 \\
0 & \eta \zeta^{*}
\end{array}\right)=\operatorname{tr}_{\left(\varphi(M) \otimes I_{H}\right)^{\prime}}\left(\eta \zeta^{*}\right) .
\end{aligned}
$$

The third assertion is [9. Lemma 2.7], and the last assertion follows from the third by taking $x=I_{H}$.

Let $E$ and $\sigma$ be fixed, now, write $d$ for $\operatorname{dim}_{l} E$, and write $P_{m}$ for the projection of $\mathcal{F}(E)$ onto $E^{\otimes m}$. Also, write $P_{\leq m}$ for the sum $\sum_{k \leq m} P_{k}$. Then it is evident from Lemma 21 that

$$
\operatorname{tr}_{\left(\varphi_{\infty}(M) \otimes I_{H}\right)^{\prime}} P_{m} \otimes I_{H}=\operatorname{dim}_{\left(\varphi_{\infty} \otimes I_{H}\right)} E^{\otimes m} \otimes_{\sigma} H=d^{m} \operatorname{dim}_{\sigma} H .
$$

Theorem 22 If $\eta \in \overline{\mathbb{D}\left(E^{\sigma}\right)}$, then:

1. If $d:=\operatorname{dim}_{l} E$ is finite, then

$$
\begin{aligned}
\kappa(\eta) & =\lim _{N \rightarrow \infty} \frac{\sum_{k=0}^{N} \operatorname{tr}_{\sigma(M)^{\prime}}\left[K(\eta)^{*}\left(P_{k} \otimes I_{H}\right) K(\eta)\right]}{\left(1+d+d^{2}+\cdots+d^{N}\right)} \\
& =\lim _{N \rightarrow \infty} \frac{\operatorname{tr}_{\left(\varphi_{\infty}(M) \otimes I_{H}\right)^{\prime}}\left[\left(P_{\leq N} \otimes I_{H}\right)\left(K(\eta) K(\eta)^{*}\right)\left(P_{\leq N} \otimes I_{H}\right)\right]}{\left(1+d+d^{2}+\cdots+d^{N}\right)} .
\end{aligned}
$$

2. If $d \geq 1$, then

$$
\begin{aligned}
\kappa(\eta)= & \lim _{N \rightarrow \infty} \frac{\operatorname{tr}_{\sigma(M)^{\prime}}\left[K(\eta)^{*}\left(P_{N} \otimes I_{H}\right) K(\eta)\right]}{d^{N}} \\
& =\lim _{N \rightarrow \infty} \frac{\operatorname{tr}_{\left(\varphi_{\infty}(M) \otimes I_{H}\right)^{\prime}}\left[\left(P_{N} \otimes I_{H}\right) K(\eta) K(\eta)^{*}\left(P_{N} \otimes I_{H}\right)\right]}{d^{N}} .
\end{aligned}
$$

3. If $d<1$, and if $\operatorname{dim}_{\sigma} H$ is finite or more generally if $\operatorname{tr}_{\sigma(M)^{\prime}}\left(I_{H}-P_{\eta}\left(I_{H}\right)\right)$ is finite, then $\operatorname{tr}_{\left(\varphi_{\infty}(M) \otimes I_{H}\right)^{\prime}}\left(K(\eta) K(\eta)^{*}\right)$ is finite and

$$
\begin{aligned}
\kappa(\eta)=(1-d) \cdot \operatorname{tr}_{\left(\varphi_{\infty}(M) \otimes I_{H}\right)^{\prime}}( & \left.K(\eta) K(\eta)^{*}\right) \\
& =(1-d) \cdot \operatorname{tr}_{\sigma(M)^{\prime}}\left(K(\eta)^{*} K(\eta)\right) .
\end{aligned}
$$

In particular, if $\|\eta\|<1$ and $d<1$, then $\kappa(\eta)=(1-d) \cdot \operatorname{dim}_{\sigma}(H)$ is independent of $\eta$. 
Proof. By definition,

$$
\kappa(\eta)=\lim _{N \rightarrow \infty} \frac{\operatorname{tr}_{\sigma(M)^{\prime}}\left(I-P_{\eta}^{N+1}\left(I_{H}\right)\right)}{\sum_{k=0}^{N} d^{k}},
$$

and by definition of $P_{\eta}$ and equation (13), the numerator in the definition of $\kappa(\eta)$ is $\sum_{k=0}^{N} \operatorname{tr}_{\sigma(M)^{\prime}}\left[K(\eta)^{*}\left(P_{k} \otimes I_{H}\right) K(\eta)\right]$. This proves the equality of the first two terms in equation (21). The equality of the third term with the first two is immediate from equation (20) in Lemma 21 (when $\mathcal{F}(E)$ is used in place of $E)$. For the second equation, write the sum $\sum_{k=0}^{N} \operatorname{tr}_{\sigma(M)^{\prime}}\left[K(\eta)^{*}\left(P_{k} \otimes I_{H}\right) K(\eta)\right]$ as $\operatorname{tr}_{\sigma(M)^{\prime}}\left[K(\eta)^{*}\left(P_{\leq N} \otimes I_{H}\right) K(\eta)\right]$, then the two numerators in equation (22) are the same by Lemma 21, But $\lim _{N \rightarrow \infty} \frac{\sum_{k=0}^{N} \operatorname{tr}_{\sigma(M)^{\prime}}\left[K(\eta)^{*}\left(P_{k} \otimes I_{H}\right) K(\eta)\right]}{\left(1+d+d^{2}+\cdots+d^{N}\right)}=$ $\lim _{N \rightarrow \infty} \frac{\operatorname{tr}_{\sigma(M)^{\prime}}\left[K(\eta)^{*}\left(P_{N} \otimes I_{H}\right) K(\eta)\right]}{d^{N}}$ and

$$
\begin{aligned}
& \lim _{N \rightarrow \infty} \frac{\operatorname{tr}_{\left(\varphi_{\infty}(M) \otimes I_{H}\right)^{\prime}}\left[\left(P_{\leq N} \otimes I_{H}\right)\left(K(\eta) K(\eta)^{*}\right)\left(P_{\leq N} \otimes I_{H}\right)\right]}{\left(1+d+d^{2}+\cdots+d^{N}\right)} \\
& =\lim _{N \rightarrow \infty} \frac{\operatorname{tr}_{\left(\varphi_{\infty}(M) \otimes I_{H}\right)^{\prime}}\left[\left(P_{N} \otimes I_{H}\right) K(\eta) K(\eta)^{*}\left(P_{N} \otimes I_{H}\right)\right]}{d^{N}}
\end{aligned}
$$

using [9, Lemma 3.2] (first noted in [16, Page 280]) and the arguments from the proof of [9, Theorem 3.3]. This proves equation (22). Finally, for equation (23), observe that when $d<1$ and $\operatorname{tr}_{\sigma(M)^{\prime}}\left(I_{H}-P_{\eta}\left(I_{H}\right)\right)<\infty$, the argument in the last paragraph of the proof of [9, Theorem 3.3] shows that the traces $\operatorname{tr}_{\sigma(M)^{\prime}}\left(I_{H}-P_{\eta}^{N}\left(I_{H}\right)\right)$ increase to a finite limit. Since each of these traces equals $\operatorname{tr}_{\left(\varphi_{\infty}(M) \otimes I_{H}\right)^{\prime}}\left[\left(P_{\leq N-1} \otimes I_{H}\right)\left(K(\eta) K(\eta)^{*}\right)\left(P_{\leq N-1} \otimes I_{H}\right)\right]$ by Lemma 21, the normality of the trace, $\operatorname{tr}_{\left(\varphi_{\infty}(M) \otimes I_{H}\right)^{\prime}}$, implies that $\operatorname{tr}_{\left(\varphi_{\infty}(M) \otimes I_{H}\right)^{\prime}}\left(K(\eta) K(\eta)^{*}\right)<$ $\infty$. As in the proof of [9, Theorem 3.3], the proof of equation (23) is immediate from the definition of $\kappa(\eta)$, the formula for the partial sums of a geometric series, and the fact that $d<1$.

Our final goal is to relate the curvature, $\kappa(\eta)$, with the trace of the "curvature operator" naturally associated to $\eta$. To define this operator, we need to say a bit more about the induced covariant representations of $E$, Definition 4 Recall that it is $\left(V, \varphi_{\infty} \otimes I_{H}\right)$, where $V: E \rightarrow B\left(\mathcal{F}(E) \otimes_{\sigma} H\right)$ is defined by the formula $V(\xi)=T_{\xi} \otimes I_{H}$. The associated map $\tilde{V}: E \otimes \mathcal{F}(E) \otimes_{\sigma} H \rightarrow \mathcal{F}(E) \otimes_{\sigma} H$ is "simply multiplication": $\tilde{V}(\xi \otimes(\eta \otimes h))=(\xi \otimes \eta) \otimes h$. As we remarked earlier, while this map looks like the identity embedding of $\sum_{k=1}^{\infty} E^{\otimes k} \otimes H$ into $\mathcal{F}(E) \otimes_{\sigma} H$, there is, in fact, a shift involved. Specifically, if $P_{k}$ is the projection of $\mathcal{F}(E)$ onto the summand $E^{\otimes k}$, then a simple calculation shows that

$$
\tilde{V}\left(I_{E} \otimes\left(P_{k} \otimes I_{H}\right)\right)=\left(P_{k+1} \otimes I_{H}\right) \tilde{V}
$$

(see [7, Corollary 2.4].) Alternatively, we may say that $\tilde{V}^{*}$ is a coisometric map in $E^{\varphi_{\infty} \otimes I_{H}}$. We shall write $\tilde{V}_{0}:=I_{\mathcal{F}(E) \otimes_{\sigma} H}$ and recursively define $\tilde{V}_{k+1}:=$ $\tilde{V}\left(I_{E} \otimes \tilde{V_{k}}\right)$. The map $\tilde{V}$ induces a non-unital endomorphism of $\left(\varphi_{\infty}(M) \otimes I_{H}\right)^{\prime}$ by the formula $\Phi_{V}(X)=\tilde{V}\left(I_{E} \otimes X\right) \tilde{V}^{*}$ and the powers of $\Phi_{V}$ are given by 
the formula $\Phi_{V}^{n}(X)=\tilde{V}_{n}\left(I_{E^{\otimes n}} \otimes X\right) \tilde{V}_{n}^{*}$ 7, Lemma 2.3] 3 We also define $\delta_{V}$ : $\left(\varphi_{\infty}(M) \otimes I_{H}\right)^{\prime} \rightarrow\left(\varphi_{\infty}(M) \otimes I_{H}\right)^{\prime}$ by the formula $\delta_{V}(X):=X-\Phi_{V}(X)$ and we define $N:=\sum_{k=0}^{\infty} d^{-k}\left(P_{k} \otimes I_{H}\right)$, where, recall, $d=\operatorname{dim}_{l} E$. Thus $\Phi_{V}$, $\delta_{V}$, and $N$ are analogues of Popescu's operators, $\phi_{S \otimes I}, d_{S \otimes I}$ and $N$, defined on pages 271 and 272 of [16]. Note that $N$ is bounded only when $d \geq 1$.

Definition 23 For $\eta \in \overline{\mathbb{D}\left(E^{\sigma}\right)}$, the curvature operator determined by $\eta$ is defined to be

$$
\delta_{V}\left[K(\eta) K(\eta)^{*}\right] N .
$$

Our goal is to prove the following analogue of [16, Theorem 2.3].

Theorem 24 If $d:=\operatorname{dim}_{l} E \geq 1$, then for $\eta \in \overline{\mathbb{D}\left(E^{\sigma}\right)}$,

$$
\kappa(\eta)=\operatorname{tr}_{\left(\varphi_{\infty}(M) \otimes I_{H}\right)^{\prime}}\left\{\delta_{V}\left[K(\eta) K(\eta)^{*}\right] N\right\} .
$$

Proof. We begin by proving an analogue of [16, Theorem 1.1]. For $Y \in$ $\left(\varphi_{\infty}(M) \otimes I_{H}\right)^{\prime}, \sum_{k=0}^{m} \Phi_{V}^{k}\left(\delta_{V}(Y)\right)=\sum_{k=0}^{m} \tilde{V}_{k}\left(I_{E^{\otimes k}} \otimes Y\right) \tilde{V}_{k}^{*}-\tilde{V}_{k+1}\left(I_{E^{\otimes k+1}} \otimes\right.$ $Y) \tilde{V}_{k+1}^{*}=Y-\tilde{V}_{m+1}\left(I_{E^{\otimes m+1}} \otimes Y\right) \tilde{V}_{m+1}^{*}$. Since $\left(V, \varphi_{\infty} \otimes I_{H}\right)$ is an induced representation in the sense of [7, Page 854], [7, Corollary 2.10] implies that the ultra-strong limit, $\lim _{n \rightarrow \infty} \tilde{V}_{n}\left(I_{E \otimes n} \otimes Y\right) \tilde{V}_{n}^{*}=0$. Thus $Y=\sum_{k=0}^{\infty} \Phi_{V}^{k}\left(\delta_{V}(Y)\right)$, where the convergence is in the ultra-strong topology. Thus for each $m \geq 0$, we have on the basis of equation (24),

$$
\begin{aligned}
& \left(P_{m} \otimes I_{H}\right) Y\left(P_{m} \otimes I_{H}\right)=\sum_{k \geq 0}\left(P_{m} \otimes I_{H}\right) \tilde{V}_{k}\left(I_{E \otimes k} \otimes \delta_{V}(Y)\right) \tilde{V}_{k}^{*}\left(P_{m} \otimes I_{H}\right) \\
& =\sum_{k \geq 0} \tilde{V}_{k}\left(I_{E \otimes k} \otimes P_{m-k} \otimes I_{H}\right)\left(I_{E \otimes k} \otimes \delta_{V}(Y)\right)\left(I_{E \otimes k} \otimes P_{m-k} \otimes I_{H}\right) \tilde{V}_{k}^{*} \\
& =\sum_{k=0}^{m} \tilde{V}_{k}\left(I_{E^{\otimes k}} \otimes\left(P_{m-k} \otimes I_{H}\right) \delta_{V}(Y)\left(P_{m-k} \otimes I_{H}\right)\right) \tilde{V}_{k}^{*} .
\end{aligned}
$$

Thus, since $\operatorname{tr}_{\left(\varphi_{\infty}(M) \otimes I_{H}\right)^{\prime}}$ restricts to $\operatorname{tr}_{\left(\varphi_{m}(M) \otimes I_{H}\right)^{\prime}}$ on $\left(P_{m} \otimes I_{H}\right)\left(\varphi_{\infty}(M) \otimes\right.$ $\left.I_{H}\right)^{\prime}\left(P_{m} \otimes I_{H}\right)$, we see that for any operator $Y$ that has finite trace calculated with respect to $\operatorname{tr}_{\left(\varphi_{\infty}(M) \otimes I_{H}\right)^{\prime}}$ and for any positive operator $Y$ in $\left(\varphi_{\infty}(M) \otimes I_{H}\right)^{\prime}$,

$$
\begin{aligned}
& \operatorname{tr}_{\left(\varphi_{\infty}(M) \otimes I_{H}\right)^{\prime}}\left(\left(P_{m} \otimes I_{H}\right) Y\left(P_{m} \otimes I_{H}\right)\right) \\
& =\sum_{k=0}^{m} \operatorname{tr}_{\left(\varphi_{m}(M) \otimes I_{H}\right)^{\prime}}\left(\tilde{V}_{k}\left(I_{E \otimes k} \otimes\left(P_{m-k} \otimes I_{H}\right) \delta_{V}(Y)\left(P_{m-k} \otimes I_{H}\right) \tilde{V}_{k}^{*}\right)\right.
\end{aligned}
$$

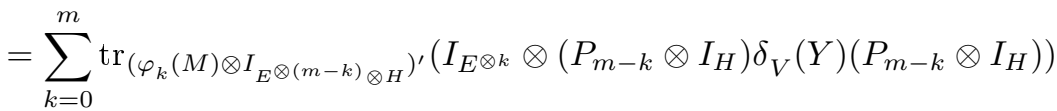

$$
\begin{aligned}
& =\sum_{k=0}^{m} \operatorname{tr}_{\left(\varphi_{m-k}(M) \otimes I_{H}\right)^{\prime}}\left(\left(P_{m-k} \otimes I_{H}\right) \delta_{V}(Y)\left(P_{m-k} \otimes I_{H}\right)\right) d^{k}
\end{aligned}
$$

\footnotetext{
${ }^{3}$ It may be helpful to keep in mind that expressions like $I_{E} \otimes_{\sigma} X$ need not represent bounded operators unless $X$ is a (bounded) operator in the commutant of $\sigma(M)$. That is why the formula for $\Phi_{V}$ does not make sense unless the argument is from $\left(\varphi_{\infty}(M) \otimes I_{H}\right)^{\prime}$.
} 
We can pass from the first line in this equation to the second and eliminate the $\tilde{V}_{k}$, since they simply identify $E^{\otimes k} \otimes\left(E^{\otimes(m-k)} \otimes H\right)$ with $E^{\otimes m} \otimes H$, and in so doing transform the trace on $\left(\varphi_{m}(M) \otimes I_{H}\right)^{\prime}, \operatorname{tr}_{\left(\varphi_{m}(M) \otimes I_{H}\right)^{\prime}}$, to the trace on

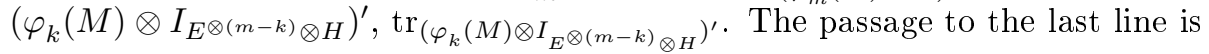
justified by part 3. of Lemma 21. Here, $\varphi_{k}$ plays the role of $\varphi$ in the lemma, while $\varphi_{m-k} \otimes I_{H}$ plays the role of $\sigma$. Also, of course, part 1. of that lemma guarantees that $\operatorname{dim}_{l} E^{\otimes k}=d^{k}$. So, if we divide the equation by $d^{m}$ and then change variables in the last sum, $m-k \rightarrow k$, we conclude that

$$
\begin{aligned}
& \frac{\operatorname{tr}_{\left(\varphi_{\infty}(M) \otimes I_{H}\right)^{\prime}}\left(\left(P_{m} \otimes I_{H}\right) Y\left(P_{m} \otimes I_{H}\right)\right)}{d^{m}} \\
& =\sum_{k=0}^{m} \operatorname{tr}_{\left(\varphi_{k}(M) \otimes I_{H}\right)^{\prime}}\left(\left(P_{k} \otimes I_{H}\right) \delta_{V}(Y)\left(P_{k} \otimes I_{H}\right)\right) d^{-k} \\
& =\sum_{k=0}^{m} \operatorname{tr}_{\left(\varphi_{\infty}(M) \otimes I_{H}\right)^{\prime}}\left(\delta_{V}(Y)\left(P_{k} \otimes I_{H}\right)\right) d^{-k} \\
& \quad=\operatorname{tr}_{\left(\varphi_{\infty}(M) \otimes I_{H}\right)^{\prime}}\left(\delta_{V}(Y)\left(\sum_{k=0}^{m}\left(P_{k} \otimes I_{H}\right) d^{-k}\right)\right) .
\end{aligned}
$$

The passage from the first line to the second simply reflects the properties of the trace and the fact that $\operatorname{tr}_{\left(\varphi_{\infty}(M) \otimes I_{H}\right)^{\prime}}$ restricts to $\operatorname{tr}_{\left(\varphi_{k}(M) \otimes I_{H}\right)^{\prime}}$ on $P_{k}\left(\varphi_{\infty}(M) \otimes\right.$ $\left.I_{H}\right)^{\prime} P_{k}$. Equation (25) is an analogue of Popescu's equation (1.4) in [16]. If $d \geq 1$, and if we replace $Y$ by $K(\eta) K(\eta)^{*}$ in equation (25), then we may take the limit as $m \rightarrow \infty$. The left hand side tends to $\kappa(\eta)$ by Theorem 22 (equation (22) ), while the right hand side tends to $\operatorname{tr}_{\left(\varphi_{\infty}(M) \otimes I_{H}\right)^{\prime}}\left\{\delta_{V}[K(\eta) K(\eta) *] N\right\}$.

\section{References}

[1] D. Alpay, P. Dewilde and H. Dym, Lossless inverse scattering and reproducing kernels for upper triangular operators, Operator Theory: Adv. and Appl. 47 (1990), 61-133.

[2] W. B. Arveson, The curvature invariant of a Hilbert module over $\mathbb{C}\left[z_{1}, \ldots, z_{d}\right]$, J. Reine Angw. Math. 522 (2000), 173-236.

[3] M. Baillet, Y. Denizeau and J.-F. Havet, Indice d'une esperance conditionelle, Comp. Math. 66 (1988), 199-236.

[4] S.D. Barreto, B.V. Bhat, V. Liebscher and M. Skeide, Type I product systems of Hilbert modules,J. Funct. Anal. 212 (2004), 121-181.

[5] K. Davidson, Free semigroup algebras. A survey in Systems, approximation, singular integral operators, and related topics (Bordeaux, 2000), 209-240, Oper. Theory Adv. Appl., 129, Birkhäuser, Basel, 2001. 
[6] P. Muhly and B. Solel, Tensor algebras over $C^{*}$-correspondences (Representations, dilations and $C^{*}$-envelopes), J. Funct. Anal. 158 (1998), 389-457.

[7] P. Muhly and B. Solel, Tensor algebras, induced representations and the Wold decomposition, Canadian J. Math. 51 (1999), 850-880.

[8] P. Muhly and B. Solel, Quantum Markov Processes (correspondences and dilations), Internat. J. Math. 13 (2002), 863-906.

[9] P. Muhly and B. Solel, The curvature and index of completely positive maps, Proc. London Math. Soc. (3) 87 (2003), 748-778.

[10] P. Muhly and B. Solel, On the Curvature of a completely positive map, Contemporary Mathematics 386 (2004), 239-251.

[11] P. Muhly and B. Solel, Hardy algebras, $W^{*}$-correspondences and interpolation theory, Math. Ann. 330 (2004), 353-415.

[12] P. Muhly and B. Solel, Canonical models for representations of Hardy algebras, Int. Eq. Oper. Theory 53 (2005), 411-452.

[13] P. Muhly and B. Solel, Hardy algebras associated with $W^{*}$-correspondences (point evaluation and Schur class functions), Operator theory, system theory and scattering theory: multidimensional generalizations, 221-241, Operator Theory: Adv. and Appl.157 (2005).

[14] P. Muhly and B. Solel, Schur Class Operator Functions and Automorphisms of Hardy Algebras, preprint arXiv:math/0606672

[15] G. Popescu, Poisson transforms on some $C^{*}$-algebras generated by isometries, J. Funct. Anal. 161 (1999), 27-61.

[16] G. Popescu, Curvature invariant over free semigroup algebras, Adv. Math. 158 (2001), 264-309.

[17] G. Popescu, Operator theory on noncommutative varieties, Indiana U. Math. J. 55 (2006), 389-442.

[18] M. Rieffel, Induce representations of $C^{*}$-algebras, Adv. Math. 13 (1974), $176-257$. 